\title{
Gold-conjugated resveratrol nanoparticles attenuate the invasion and MMP-9 and COX-2 expression in breast cancer cells
}

\author{
SUN YOUNG PARK ${ }^{2}$, SEON YEONG CHAE $^{1}$, JIN OH PARK $^{1}$, KYU JIN LEE $^{1}$ and GEUNTAE PARK ${ }^{1,2}$ \\ ${ }^{1}$ Department of Nano Fusion Technology, Graduate School, Pusan National University, Busan 609-735; \\ ${ }^{2}$ Bio-IT Fusion Technology Research Institute, Pusan National University, Busan 609-735, Republic of Korea
}

Received December 4, 2015; Accepted January 26, 2016

DOI: $10.3892 /$ or.2016.4716

\begin{abstract}
Gold nanoparticles (AuNPs) with antitumorigenic effects obstruct the initiation, development and progression of tumors via the regulation of various processes, such as proliferation and apoptosis. However, the effects of AuNPs on breast cancer metastasis have not been well studied, and their response to $12-O$-tetradecanoylphorbol-13-acetate (TPA) stimulation remains unclear. Therefore, we synthesized resveratrol-capped gold nanoparticles (Rev-AuNPs) using green nanotechnology and investigated their potential anti-invasive properties in human breast cancer cells in response to TPA stimulation. The Rev-AuNPs formed spherical nanoparticles of $22.28 \pm 2.98 \mathrm{~nm}$ in diameter. Next, we found that non-cytotoxic concentrations of Rev-AuNPs significantly suppressed the TPA-induced migration and invasion abilities of breast cancer cells. Rev-AuNPs suppressed TPA-induced enzymatic activity and the expression of matrix metalloproteinase (MMP)-9 and cyclooxygenase-2 (COX-2). Furthermore, Rev-AuNP treatment remarkably downregulated TPA-induced nuclear translocation and transcriptional activation of nuclear transcription factor- $\kappa \mathrm{B}(\mathrm{NF}-\kappa \mathrm{B})$ and activator protein-1 (AP-1). Rev-AuNPs reduced the phosphorylation of phosphoinositide 3-kinase/Akt (PI3K/Akt) and extracellular signal-regulated kinase (ERK) $1 / 2$ signaling, but did not affect the phosphorylation of Jun N-terminal kinase (JNK) or p38 MAPK in the TPA-stimulated breast cancer cells. Notably, Rev-AuNPs generally showed better anti-invasive activity than resveratrol without cytotoxicity. The inhibitory effect of Rev-AuNPs on MMP-9, COX-2, NF-kB, AP-1, PI3K/Akt and ERK activation was stronger than that of resveratrol for the same concentrations. We also demonstrated that Rev-AuNPs induced heme oxygenase-1 (HO-1) expression and that the inhibition of MMP-9 and COX-2 expression and MMP-9 enzymatic activity of Rev-AuNPs were abrogated by siRNA knockdown of $\mathrm{HO}-\mathrm{I}$ expression. Our findings revealed that the anti-invasive effects
\end{abstract}

Correspondence to: Professor Geuntae Park, Department of Nano Fusion Technology, Graduate School, Pusan National University, Busan 609-735, Republic of Korea

E-mail: gtpark@pusan.ac.kr

Key words: gold nanoparticles, resveratrol, invasion, TPA, MMP-9 of Rev-AuNPs in response to TPA-stimulation were mediated by the suppression of MMP-9, COX-2, NF- $\mathrm{B}, \mathrm{AP}-1, \mathrm{PI} 3 \mathrm{~K} / \mathrm{Akt}$ and ERK and/or the activation of HO-1 signaling cascades. This novel finding emphasizes the pharmacological ability of Rev-AuNPs to treat breast cancer metastasis.

\section{Introduction}

Epidemiological surveys have shown that breast cancer is the most common malignant cancer in females. The process of tumor progression and metastasis is multipart and evolutionary, involving numerous phases and events at the cellular and molecular levels. Tumor cells need to detach from each other, migrate, pierce through vasculature and establish angiogenesis (1). Metastasis is one of the leading causes of breast cancer-related mortality among females. It is well known that metastasis and invasion are basic properties of breast cancer cells and the main cause of cancer-related deaths. Several studies have reported that the invasion and metastasis of breast cancer involve multiple biological processes, including cell proliferation, motility, adhesion, degradation of the basement membrane, invasion and metastasis $(2,3)$. Therefore, efforts to develop additional breast cancer strategies for the treatment and diagnosis of breast cancer are of great importance.

Extravasation from the vessels is mediated by the action of extracellular proteases, among which the matrix metalloproteinases (MMPs) play crucial roles. It is well established that MMPs, which are vital enzymes that degrade the extracellular matrix, play central roles in tumor invasion and metastasis. As a main member of the MMPs, upregulation of the activity and expression of MMP-9 is frequently observed in breast cancers with invasive and metastatic capability (3). The induction of cyclooxygenase-2 (COX-2) is a characteristic feature of the tumor environment; it leads to invasiveness, angiogenesis, and metastatic potential. COX-2 is overexpressed in breast cancer cell lines and plays an important role in breast cancer invasion (4). MMP-9 and COX-2 are constitutively activated in most cancers and offer effective targets for therapeutic development. MMP-9 and COX-2 are involved in tumor progression via a wide variety of signaling molecules and mechanisms. Furthermore, the transcription factors nuclear transcription factor $-\kappa \mathrm{B}(\mathrm{NF}-\kappa \mathrm{B})$ and activator protein-1 (AP-1) which are direct targets of MMP-9 and COX-2, have been correlated with increased metastatic potential in cancer. Recent studies 
have demonstrated that NF- $\mathrm{KB}$ and AP-1 promote the migration and invasion of cancer cells via the regulation of a number of genes $(5,6)$.

Resveratrol is a natural dietary phytochemical that has a preventive effect against breast cancer. It is an active ingredient in grapes, wine, mulberries, peanuts and other vegetation. Resveratrol has antioxidant, anti-inflammatory, and antisenescence properties, and its potential as an anticancer drug is currently being evaluated in clinical trials for various types of cancers $(7,8)$. Its anticancer ability is the key to its therapeutic potential. It inhibits cell growth, tumor proliferation, invasion, metastasis and angiogenesis by downregulating many molecular signaling targets, including MMP-9, COX-2, NF- $\kappa$ B and AP-1 (7,9). However, the well-known clinical applications of resveratrol in cancer have been limited owing to its poor bioavailability. Therefore, it is important to make resveratrol in more effective forms with respect to efficacy and stability $(10,11)$.

Nanoparticle research has comprehensively increased in recent years to include applications in areas such as advanced nanomedicine. Moreover, nanotechnology has seen remarkable improvements and has potential for medicinal applications, including drug development and delivery. Recently, gold nanoparticles (AuNPs) are used extensively as compounds for pharmaceutical products targeting several diseases, including cancer, neurodegenerative disease and hepatitis, owing to their unfamiliar optoelectronic and physicochemical properties $(12,13)$. Therefore, the biological synthesis of AuNPs is a focus of current research. AuNPs are considered more eco-friendly and cost-effective than other chemical and physical methods owing to the reduced use of hazardous reagents and solvents, improved material and energy efficiency from the chemical process, and enhanced design of non-toxic products $(14,15)$. Phytochemicals have been used recently to biologically synthesize AuNPs. The nanoformulation of phytochemicals shows enhanced cellular uptake, bioavailability, and anticancer activity as compared to phytochemicals (16). Although AuNPs have been shown to exert antitumor effects in various models, only a few studies have reported the antitumor properties of AuNPs and the mechanism by which AuNPs interact with human breast cancer cells. A recent study found that AuNPs in the 50-300 nm size range caused significant time- and dose-dependent toxicity in human breast cancer MCF-7 cells through the reactive oxygen species (ROS)-/Jun N-terminal kinase (JNK)-mediated mitochondrial pathway (17-19). However, it is still unclear whether AuNPs have a direct beneficial effect on breast cancer metastasis beside the effects involving various molecular targets. In the present study, we demonstrated the biological synthesis of AuNPs using resveratrol, which acts as a reducing and stabilizing agent, and investigated the anti-invasive effect of resveratrol-capped gold nanoparticles (Rev-AuNPs) on 12-O-tetradecanoylphorbol-13-acetate (TPA)-induced breast cancer cells, with a focus on the key molecular events involved. Rev-AuNP treatment resulted in inhibitory effects on TPA-induced migration and invasion as well as the activation of multiple gene products linked to MMP-9, COX-2, NF- $\kappa \mathrm{B}$, AP-1, phosphoinositide 3-kinase/Akt (PI3K/Akt) and extracellular signal-regulated kinase (ERK) in human breast cancer cells. In addition to the key events in breast cancer progres- sion, the crucial role of the induction of heme oxygenase-1 (HO-1) in response to Rev-AuNP treatment was investigated in TPA-stimulated breast cancer cells.

\section{Materials and methods}

Materials and reagents. Resveratrol and other chemicals were purchased from Sigma-Aldrich (St. Louis, MO, USA).

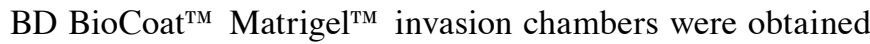
from BD Biosciences (San Jose, CA, USA). Antibodies against phosphorylated p38 (p-p38), p-JNK, p-ERK, MMP-2 and MMP-9 were purchased from Cell Signaling Technology (Beverly, MA, USA). HO- 1 small interfering RNA (siRNA), and antibodies against COX-1, COX-2, ERK, JNK, p38, c-Jun, c-Fos and NF- $\kappa$ B and TBP were purchased from Santa Cruz Biotechnology (Santa Cruz,CA, USA). Cell culture RPMI-1640 medium and fetal bovine serum (FBS) were purchased from Gibco-BRL Invitrogen (Carlsbad, CA, USA). FuGENE6 and $\mathrm{X}$-tremeGENE siRNA transfection reagents were purchased from Roche (Indianapolis, IN, USA).

Green synthesis and characterization of Rev-AuNPs. Rev-AuNPs were prepared by the reduction method using chloroauric acid according to a previous study (20), with slight modifications. In the synthesis protocol, resveratrol $(500 \mathrm{mM})$ in dimethyl sulfoxide (DMSO) was added to a stirred $\mathrm{HAuCl}_{4}$ solution $(1 \mathrm{mM})$, and kept at room temperature for $10 \mathrm{~min}$. The color change from yellow to deep ruby red indicated the formation of gold nanoparticles. The mixture was stirred for another $10 \mathrm{~min}$. The obtained gold nanoparticles are stable for $>1$ year. The UV-visible spectrum of the sampled resveratrol or Rev-AuNP solution was measured using an Ultrospec 6300 Pro (GE Healthcare Life Sciences, Buckinghamshire, UK) at wavelengths in the $300-800 \mathrm{~nm}$ range. Average particle sizes and zeta potentials of different formulations were determined by a dynamic light scattering (DLS) method using Data Transfer Assistance (DTA) software and a Zetasizer Nano ZS90 (Malvern Instruments Ltd., Malvern, UK). The morphology and distribution of the Rev-AuNPs were examined using a transmission electron microscope (TEM; Hitachi, Tokyo, Japan).

Cell culture. The human breast cell line MCF-7 was obtained from the American Type Culture Collection (ATCC; Manassas, VA, USA). Cells were grown in RPMI-1640 medium supplemented with $10 \%$ heat-inactivated FBS and $1 \%$ penicillin-streptomycin at $37^{\circ} \mathrm{C}$ in a humidified incubator with a $5 \% \mathrm{CO}_{2}$ atmosphere.

Cell invasion assay. A cell invasion assay was conducted using BioCoat $^{\mathrm{TM}}$ Matrigel $^{\mathrm{TM}}$ invasion chambers according to the manufacturer's instructions. Briefly, the Matrigel coating was re-hydrated in $0.5 \mathrm{ml}$ of Dulbecco's modified Eagle's medium (DMEM) for $30 \mathrm{~min}$ immediately before the experiments. Cells $\left(5 \times 10^{4}\right)$ suspended in $0.5 \mathrm{ml}$ of serum-free medium were added to the upper chamber of the Matrigel-coated filter inserts. After treatment with sanguinarine for $1 \mathrm{~h}, 0.5 \mathrm{ml}$ of serum-free medium containing $50 \mathrm{nM}$ TPA was added to the bottom well as a chemoattractant. The chambers were then incubated for $24 \mathrm{~h}$. After incubation, cells on the upper side 
of the chamber were removed using cotton swabs, and cells that had migrated were fixed and stained with $2 \%$ ethanol containing $0.2 \%$ crystal violet powder. Invading cells were enumerated under a light microscope at a magnification of $\mathrm{x} 10$.

In vitro wound healing repair assay. For the in vitro wound healing repair assay (cell migration assay), the cells were seeded in a $24-$ well culture dish until reaching $90 \%$ confluency. The cells were then maintained in serum-free medium for $12 \mathrm{~h}$. The monolayers were carefully scratched using a $200-\mu 1$ pipette tip. Cellular debris was removed by washing with phosphate-buffered saline, after which the cells were incubated in serum-free medium. The migrated cells were then fixed with cold $75 \%$ methanol for 30 min and washed 3 times with phosphate-buffered saline. The cultures were photographed at 0 and $24 \mathrm{~h}$ to monitor the migration of cells into the wounded area, after which the closure of the wounded area was calculated.

Gelatin zymography assay. The enzyme activities of MMP-2 and MMP-9 in conditioned medium were determined using a gelatin zymography protease assay. Briefly, cells $\left(2 \times 10^{5}\right)$ were seeded into 6-well plates and allowed to grow to $80 \%$ confluency. The cells were then maintained in serum-free medium for $12 \mathrm{~h}$ prior to treatments with sanguinarine and TPA for $24 \mathrm{~h}$. Conditioned media were collected, cleared by centrifugation, and mixed with 2X SDS sample buffer (Invitrogen), followed by electrophoresis in a polyacrylamide gel containing $0.1 \%$ (w/v) gelatin. Following electrophoresis, the gels were incubated in renaturing buffer (2.5\% Triton X-100) with gentle agitation to remove SDS, followed by incubation in developing buffer (50 mM Tris- $\mathrm{HCl}$ buffer, $\mathrm{pH} 7.4$ and $10 \mathrm{mM} \mathrm{CaCl}_{2}$ ) overnight at $37^{\circ} \mathrm{C}$ to allow digestion of the gelatin. Gels were then stained with SimplyBlue SafeStain (Invitrogen) until clear bands suggestive of gelatin digestion were visible.

Western blot analysis. Cells were harvested in ice-cold lysis buffer consisting of $1 \%$ Triton X-100,1\% deoxycholate and $0.1 \%$ SDS. The protein content of the cell lysates was then determined using the Bradford reagent (Bio-Rad Laboratories, Hercules, CA, USA). Proteins in each sample (50 $\mu \mathrm{g}$ of total proteins) were resolved by $12 \%$ SDS-PAGE, transferred to a polyvinylidene difluoride membrane, and exposed to the appropriate antibodies. The proteins were visualized by enhanced chemiluminescence detection (Amersham Biosciences, Piscataway, NJ, USA) using horseradish peroxidase-conjugated anti-rabbit or anti-mouse secondary antibodies. Images were captured using an ImageQuant 350 analyzer (Amersham Biosciences).

Real-time PCR. Total cellular RNA was isolated using an RNA Spin Mini RNA Isolation kit (GE Healthcare) according to the manufacturer's instructions. One microgram of total RNA was reverse-transcribed using Maxime RT PreMix (Intron Biotechnology, Seongnam, Korea) and anchored oligo$\mathrm{dT}_{15}$ primers. Real-time PCR was performed with SYBR-Green Master Mix (Applied Biosystems, Foster City, CA, USA) using a Chromo4 instrument (Bio-Rad). The relative amount of target mRNA was determined using the $\mathrm{Ct}$ method by normalizing target mRNA Ct values to those for $G A P D H(\triangle \mathrm{Ct})$. The real-time PCR cycling conditions were $95^{\circ} \mathrm{C}$ for $5 \mathrm{~min}$,
40 cycles for $30 \mathrm{sec}$ at $95^{\circ} \mathrm{C}, 20 \mathrm{sec}$ at $55^{\circ} \mathrm{C}$ and $30 \mathrm{sec}$ at $72^{\circ} \mathrm{C}$, followed by fluorescence measurement. The primer sequences used were as follows: MMP-9 sense (5'-ttccetggagacctgaga acc-3') and MMP-9 antisense (5'-cggcaagtcttccgagtagtttt3'); COX-2 sense (5'-tacaagcagtggcaaaggc-3') and COX-2 antisense (5'-agatcatctctgcetgagtatctt-3'); GAPDH sense (5'-aggtggtctcct ctgacttc-3') and GAPDH antisense (5'-taccaggaaatgagcttgac-3').

Transient transfection and dual-luciferase assay. To determine the promoter activity, we used a Dual-Luciferase Reporter Assay system (Promega, Madison, WI, USA). Cells were transfected with the NF-кB or AP-1 luciferase reporter plasmids (Stratagene, Grand Island, NY, USA) using the FuGENE6 reagent (Roche Applied Science, Basel, Switzerland) according to the manufacturer's instructions. Renilla luciferase control plasmid pRL-CMV (Promega) was co-transfected as an internal control to determine the transfection efficiency. Twenty-four hours after transfection, cells were incubated with the indicated reagents for $1 \mathrm{~h}$, and then treated with TPA for $24 \mathrm{~h}$. The luciferase activity was assayed with a Dual-Luciferase Assay kit according to the manufacturer's instructions. Luminescence was measured with a GloMax ${ }^{\mathrm{TM}} 96$ Microplate Luminometer (Promega).

Transient transfection of siRNA. Transfection of MCF-7 cells with siRNA was performed using the X-tremeGENE siRNA transfection reagent (Roche Applied Science), according to the manufacturer's instructions. Commercially available human HO-1-specific siRNAs and negative control siRNAs were used for transfection. Briefly, X-tremeGENE siRNA transfection reagent $(10 \mu \mathrm{l})$ was added to $100 \mu \mathrm{l}$ of serum-free medium containing $2 \mu \mathrm{g}$ of each siRNA oligo, and the mixture was incubated for $20 \mathrm{~min}$ at room temperature.

Statistical analysis. Each experiment was repeated at least 3 times, and all results are expressed as means \pm SE. Statistical analysis was performed using SPSS (version 18.0) to determine significant differences among groups. One-way analyses of variance (ANOVA) followed by Tukey's post hoc tests were used for comparisons between 3 or more groups. Differences with $\mathrm{P}<0.05$ were considered statistically significant.

\section{Results}

Characterization of the Rev-AuNPs. When resveratrol was used as a reductant, the Rev-AuNPs turned pink in color and exhibited a characteristic surface plasmon resonance band with maximum absorbance at $540 \mathrm{~nm}$ (Fig. 1A). Rev-AuNPs were characterized based on their size, distribution and zeta potential using DLS. Based on the DLS analysis, the Rev-AuNPs had a diameter of $30.75 \pm 3.41 \mathrm{~nm}$ and a zeta potential of $-32.8 \pm 2.47 \mathrm{mV}$ (Fig. 1B). The obtained Rev-AuNPs were visualized by TEM and were predominantly spherical in shape $(22.28 \pm 2.98 \mathrm{~nm})$. The images revealed that the Rev-AuNPs were well dispersed without any aggregation, suggesting that resveratrol acted as both a reductant and a stabilizing agent (Fig. 1C). The cytotoxic effect of Rev-AuNPs on MCF-7 breast cancer cells was determined. Rev-AuNPs at a concentration of $10 \mu \mathrm{M}$ were non-toxic to MCF-7 breast cancer cells (Fig. 1D). Therefore, for all experiments, we used $<10 \mu \mathrm{M}$ 
A
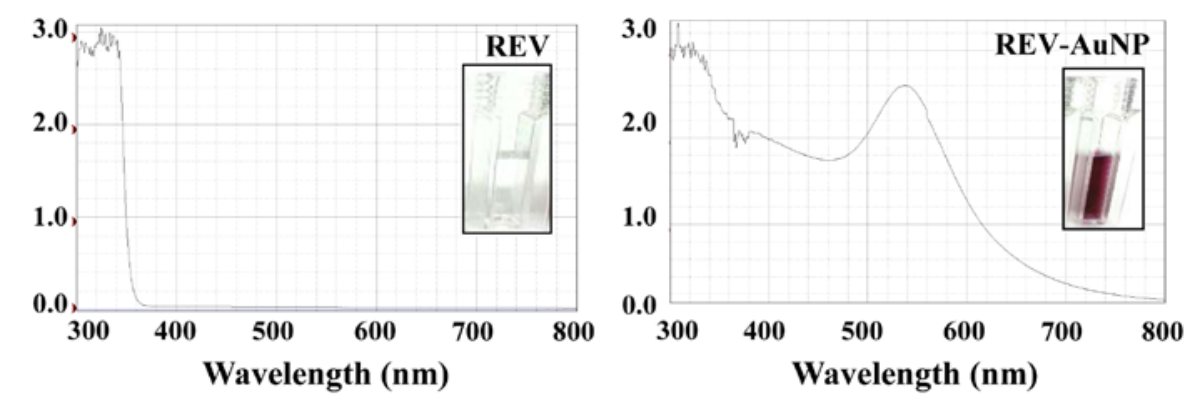

B
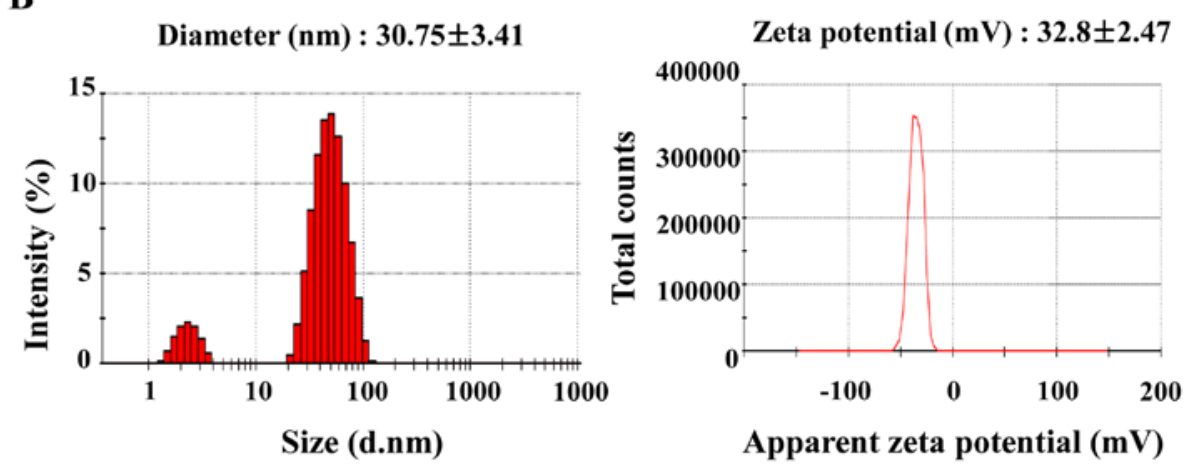

C

D
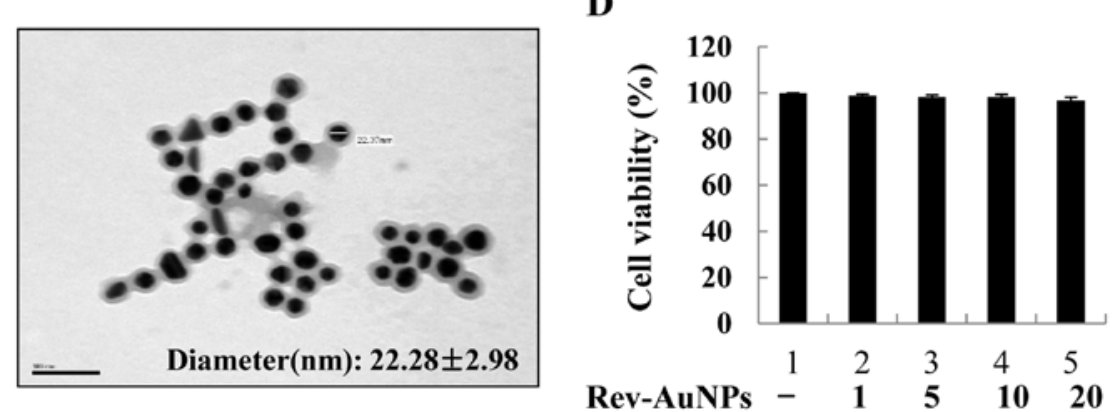

Figure 1. Synthesis and characterization of gold-conjugated resveratrol (Rev) nanoparticles. (A) Representative UV-vis spectroscopy of prepared Rev and gold-conjugated resveratrol nanoparticles (Rev-AuNPs). (B) Particle size and zeta potential of Rev-AuNPs determined by dynamic light scattering (DLS). (C) Representative transmission electron microscopy (TEM) image of Rev-AuNPs. (D) Effect of Rev-AuNPs on cell viability. Cells were treated with the indicated concentration of Rev-AuNPs in the presence of TPA $(50 \mathrm{ng} / \mathrm{ml})$ for $24 \mathrm{~h}$. Cell viabilities were determined by the MTT assay.

Rev-AuNPs and determined their anti-invasive properties in TPA-stimulated MCF-7 breast cancer cells.

Effect of Rev-AuNP on TPA-induced breast cancer cell migration and invasion. Breast cancer cell migration and invasion through the basement membrane are important steps in breast cancer malignancy. Since proteolytic digestion of the extracellular matrix and migration of cancer cells across the blood vessel-lining endothelial monolayers play crucial roles in the metastasis process (2), we investigated the effects of Rev-AuNPs on the migration and invasion of MCF-7 breast cancer cells and compared the observed effects to those of resveratrol. The effect of Rev-AuNPs on MCF-7 breast cancer cell migration and invasion was determined by a wound healing assay with or without TPA stimulation for $24 \mathrm{~h}$. We found that TPA-induced MCF-7 breast cancer cell migration was significantly inhibited by the Rev-AuNPs (Fig. 2A). The invasive ability of the MCF-7 breast cancer cells was determined by a Matrigel Transwell invasion assay. TPA treatment significantly promoted the invasiveness of the MCF-7 breast cancer cells compared to that observed in the untreated cells. However, Rev-AuNP pretreatment significantly inhibited TPA-induced invasion of the MCF-7 breast cancer cells (Fig. 2B). Notably, the inhibitory effect of Rev-AuNPs on migration and invasion was stronger that of resveratrol at the same concentration (Fig. 2C).

Effect of Rev-AuNP on TPA-induced MMP-9 and COX-2 activation in breast cancer cells. MMP-9 and gelatinase are considered to be primarily responsible for the degradation of major extracellular matrix components and subsequent metastasis through the basement membrane (21). Therefore, gelatin zymography, western blotting and real-time PCR were performed to detect changes in MMP-9 activity, protein and mRNA expression levels in the TPA-stimulated MCF-7 breast cancer cells. MMP-9 activity as well as protein and mRNA expression were predominantly upregulated upon TPA stimulation. Nevertheless, pretreatment with Rev-AuNPs suppressed TPA-induced over-activation of MMP-9 activity, protein and mRNA expression (Fig. 3A and B). COX-2 is observed in many 
A
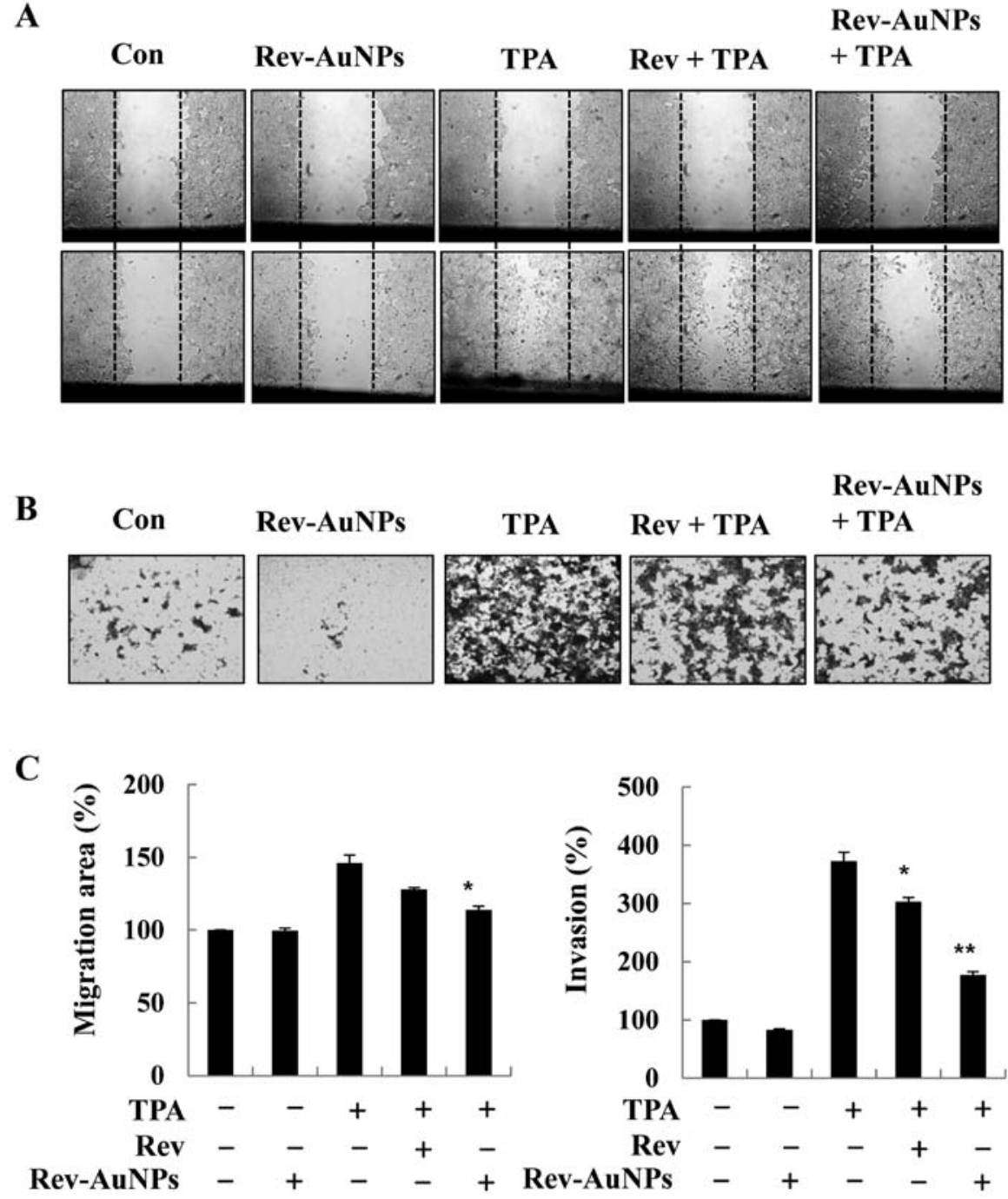

Figure 2. Gold-conjugated resveratrol nanoparticles (Rev-AuNPs) inhibit TPA-induced migration and invasion of human breast cancer cells. (A) MCF-7 cells were scratched with a pipette tip, pretreated with resveratrol (Rev) $(10 \mu \mathrm{M})$ or Rev-AuNPs $(10 \mu \mathrm{M})$, and then treated with $12-O$-tetradecanoylphorbol-13-acetate (TPA; $50 \mathrm{ng} / \mathrm{ml}$ ) for $24 \mathrm{~h}$. Migrating cells were analyzed by phase contrast microscopy. (B) Cells were treated with Rev-AuNPs (10 $\mu$ M), followed by TPA $(50 \mathrm{ng} / \mathrm{ml})$ treatment for $24 \mathrm{~h}$. Cells that invaded the bottom side of the filter were stained with crystal violet and counted under a microscope. (C) Quantified levels of cell migration and invasion expressed relative to the basal invasion of untreated cells. Each bar represents the mean \pm SE of 3 independent experiments in each group. ${ }^{*} \mathrm{P}<0.05,{ }^{* *} \mathrm{P}<0.01$ significant compared to cells treated with TPA alone.

types of cancer and has been shown to stimulate metastasis. We explored the inhibitory activity of Rev-AuNPs on TPA-induced COX-2 expression at the protein and mRNA levels using western blotting and real-time PCR. The TPA-induced COX-2 mRNA and protein expression levels were also significantly attenuated by the Rev-AuNPs (Fig. 3A and C). It was interesting to observe that the inhibitory effect of Rev-AuNPs on the activation of MMP-9 and COX-2 was stronger than that of resveratrol at the same concentration. Taken together, these results indicated that the inhibition of MMP-9 and COX-2 activation by Rev-AuNPs was responsible for the suppression of migration and invasion in the TPA-stimulated MCF-7 breast cancer cells.

Effect of Rev-AuNP on TPA-induced NF- $\kappa B$ and AP-1 activation in breast cancer cells. NF- $\kappa \mathrm{B}$ and $\mathrm{AP}-1$ are essential regulators of MMP-9 and COX-2, which are critically involved in cancer metastasis. Hence, we observed the effects of Rev-AuNPs on NF- $\mathrm{B}$ and AP-1 signaling in activated breast cancer cells (6). After the cells were treated with the Rev-AuNPs and TPA for $30 \mathrm{~min}$, we used western blotting to examine the phosphorylation and nuclear translocation of $\mathrm{NF}-\kappa \mathrm{B}$ subunit p65 in the nuclear extract. The phosphorylation and nuclear translocation of $N F-\kappa B$ subunit p65 were significantly enhanced after cells were challenged with TPA. Treatment with Rev-AuNPs apparently inhibited the over-activation of $N F-\kappa B$ subunit p65. Similarly, the nuclear translocation levels of AP-1 subunit c-Jun and c-fos were significantly attenuated by Rev-AuNPs in comparison with the TPA-induced cells. However, Rev-AuNPs had no obvious effect on the activation of the NF- $\kappa B$ subunit $p 65$ or AP-1 (Fig. 4A). To confirm the effect of Rev-AuNPs on the transcriptional activity of NF- $\kappa \mathrm{B}$ and AP-1, we used a luciferase reporter assay containing the $\mathrm{NF}-\kappa \mathrm{B}$ and AP-1 binding regions. Treatment with TPA-stimulated MCF-7 breast cancer cells increased the promoter activity of NF- $\kappa \mathrm{B}$ and AP-1; treatment with the Rev-AuNPs decreased the TPA-stimulated promoter activity of NF- $\mathrm{B}$ and AP-1 (Fig. 4B and C). 

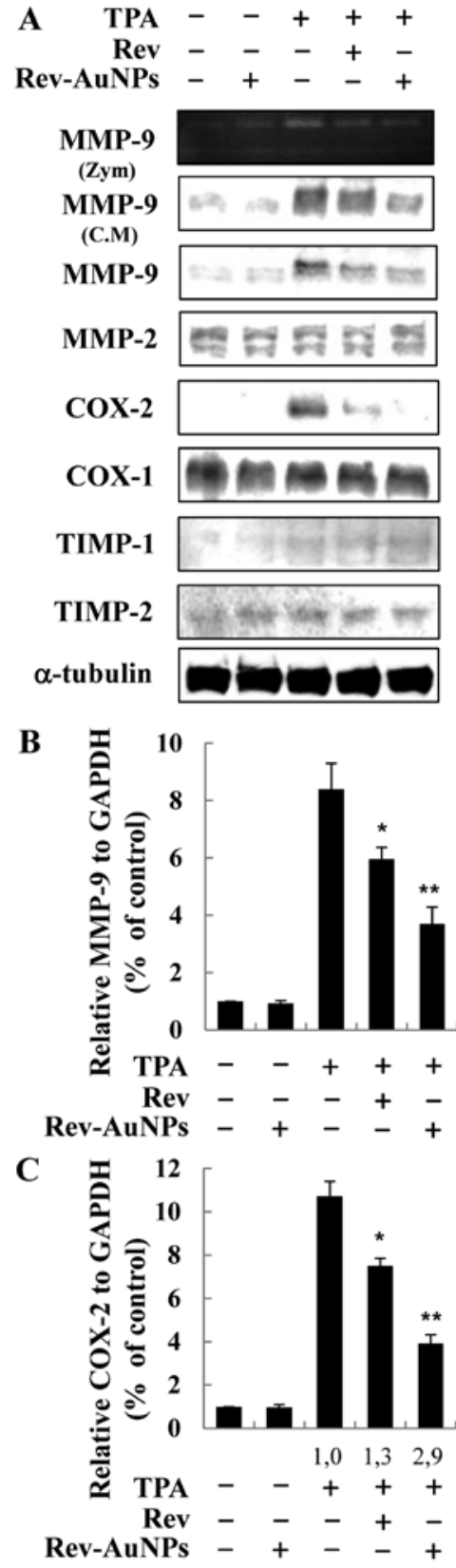

Figure 3. Effects of gold-conjugated resveratrol nanoparticles (Rev-AuNPs) on MMP-9, MMP-2, COX-1, COX-2, TIMP-1 and TIMP-2 expression in TPA-induced human breast cancer cells. (A) MCF-7 cells were treated with resveratrol (Rev) $(10 \mu \mathrm{M})$ or Rev-AuNPs $(10 \mu \mathrm{M})$ or $1 \mathrm{~h}$, followed by TPA treatment $(50 \mathrm{ng} / \mathrm{ml})$ for $24 \mathrm{~h}$. MMP-9 enzymatic activity was analyzed by gelatin zymography (Zym), secretion by western blotting and intracellular protein expression by western blotting. The protein levels of MMP-9, MMP-2, COX-1, COX-2, TIMP-1 and TIMP-2 were evaluated by western blotting. $\mathrm{CM}$, conditioned medium. The relative $M M P-9$ (B) and $C O X-2$ (C) mRNA expression levels $\left(2^{-\Delta C}\right)$ were quantified by real-time PCR and calculated by subtracting the $C t$ value for $G A P D H$ from the $C t$ value for $M M P-9$ and $C O X-2$, which were determined by real-time RT-PCR: $\triangle C \mathrm{t}=C \mathrm{t}(M M P-9$ or $C O X-2)-C t(G A P D H)$. Quantitative analyses of $M M P-9$ and $C O X-2$ mRNA expression are expressed as the means $\pm \mathrm{SE}$ of 3 independent experiments in each group. ${ }^{*} \mathrm{P}<0.05,{ }^{* *} \mathrm{P}<0.01$ significant compared to cells treated with TPA alone.

Effect of Rev-AuNP on TPA-induced activation of Akt and ERK in breast cancer cells. PI3K/Akt and mitogen-activated protein kinases (MAPKs) are important for the expression of both MMP-9 and COX-2. They act as specific targets in cancer metastasis (4). We examined whether PI3K/Akt and MAPK
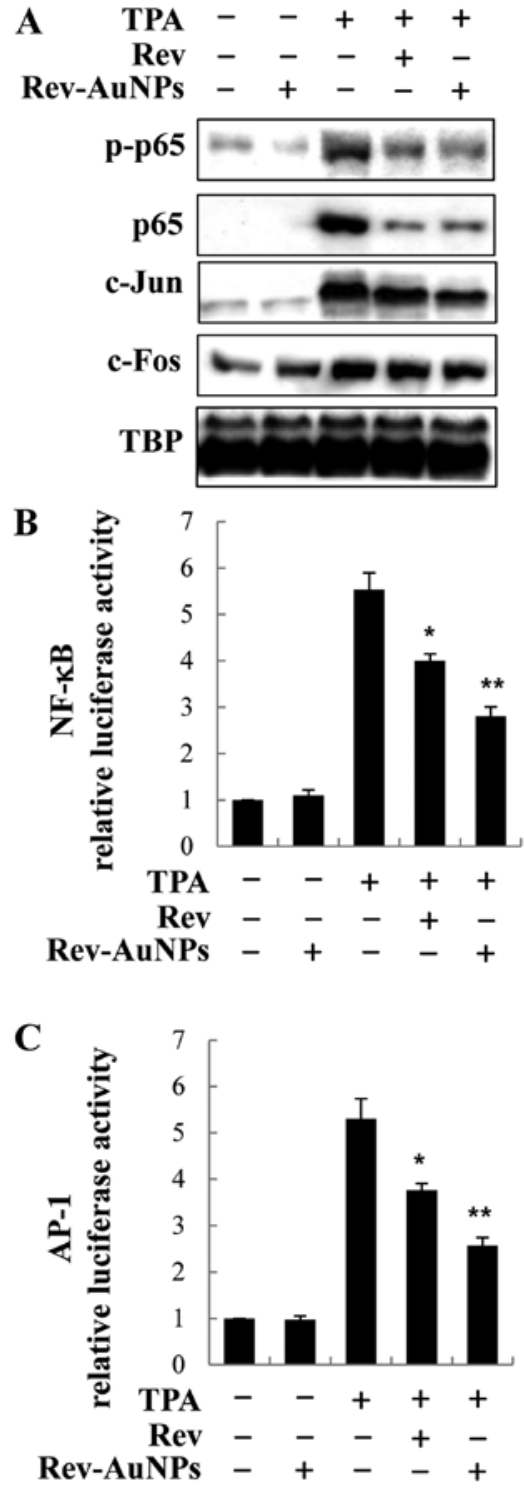

Figure 4. Gold-conjugated resveratrol nanoparticles (Rev-AuNPs) downregulate NF- $\mathrm{B}$ and AP-1 activation in TPA-induced breast cancer cells. (A) MCF-7 cells were treated with $\operatorname{Rev}(10 \mu \mathrm{M})$ or Rev-AuNPs $(10 \mu \mathrm{M})$ for $1 \mathrm{~h}$, followed by TPA $(50 \mathrm{ng} / \mathrm{ml})$ treatment for $1 \mathrm{~h}$. Nuclear extracts were prepared and analyzed by western blotting using anti-NF- $\kappa \mathrm{B}$ p65, c-Jun and c-Fos antibodies. (B and C) Cells were transfected with an NF- $\kappa$ B or AP-1 luciferase reporter. After $24 \mathrm{~h}$, the cells were incubated with Rev $(10 \mu \mathrm{M})$ or Rev-AuNPs $(10 \mu \mathrm{M})$ for $1 \mathrm{~h}$, followed by stimulation with TPA $(50 \mathrm{ng} / \mathrm{ml})$ for $24 \mathrm{~h}$. Equal amounts of cell extracts were assayed for dual-luciferase activity. Expression of the Renilla luciferase control was used to normalize the NF- $\kappa \mathrm{B}$ or AP-1 luciferase activity. Each bar represents the mean \pm SE of 3 independent experiments in each group. ${ }^{*} \mathrm{P}<0.05,{ }^{* *} \mathrm{P}<0.01$ significant compared to cells treated with TPA alone.

activity was inhibited via treatment with the Rev-AuNPs. We assessed the phosphorylation levels of PI3K/Akt and MAPKs, including Akt, JNK, p38 and ERK. When cells were stimulated with TPA, the levels of phosphorylated Akt, JNK, p38 and ERK were significantly increased. As shown in Fig. 5, the phosphorylation of Akt and ERK was markedly inhibited by the Rev-AuNPs. By contrast, Rev-AuNP pretreatment did not significantly effect TPA-induced JNK and p38 phosphorylation. These results demonstrated that Rev-AuNPs were able to reduce MMP- 9 and COX-2 activation by inhibiting the activation of the Akt and ERK signaling pathway. 


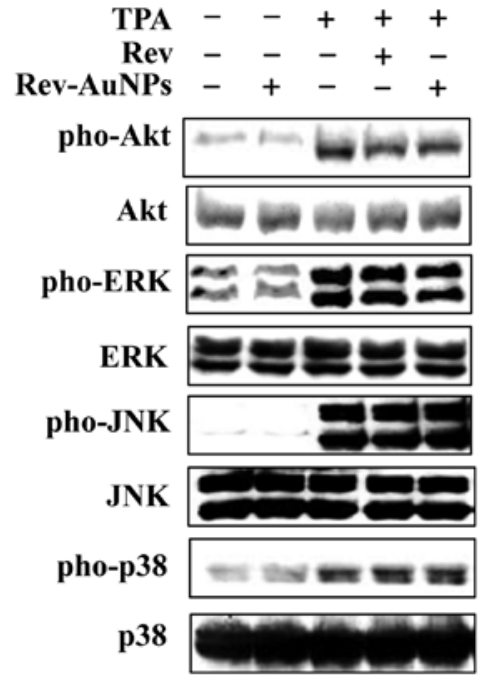

Figure 5. Effect of the gold-conjugated resveratrol nanoparticles (RevAuNPs) on TPA-induced activation of the Akt and MAPK signaling pathways. MCF-7 cells were treated with resveratrol (Rev) $(10 \mu \mathrm{M})$ or RevAuNPs $(10 \mu \mathrm{M})$ for $1 \mathrm{~h}$, followed by stimulation with TPA $(50 \mathrm{ng} / \mathrm{ml})$ for $1 \mathrm{~h}$. Equal amounts of cell extract were analyzed by western blotting with anti-phospho-Akt, ERK1/2, JNK and p38 antibodies.

Inhibitory effect of Rev-AuNPs on TPA-induced MMP-9 activity via $\mathrm{HO}-1$. We hypothesized that the upregulation of HO- 1 by Rev-AuNPs may be a key factor in the attenuation of the TPA-induced invasion response. Accordingly, we determined the effect of Rev-AuNPs on the expression of HO-1 in MCF-7 breast cancer cells. We found quite interesting results; Rev-AuNP treatment gradually upregulated the expression of HO-1 at the mRNA and protein levels in a dose-dependent manner (Fig. 6A and B). Numerous anticancer drug candidates prevent tumor progression via the induction of HO-1. Therefore, we characterized the Rev-AuNP-mediated antiinvasion effects in response to TPA stimulation with respect to the overexpression of $\mathrm{HO}-\mathrm{l}$. We established an $\mathrm{HO}-\mathrm{l}$ knockdown model by transfection of $H O-1$ siRNA to cells, which definitely inhibit HO-1 expression. The effective knockdown of HO-1 was confirmed using a western blot assay. Next, we observed that TPA-induced increases in MMP-9 activity and protein expression were suppressed by Rev-AuNPs, but this effect was limited in the $\mathrm{HO}-1$-knockdown cells (Fig. 6C). Our findings strongly support the notion that the Rev-AuNP antiinvasive effect was associated with the activation of HO-1 in the MCF-7 breast cancer cells.

\section{Discussion}

Resveratrol has long been part of the daily diet worldwide and has not been shown to cause any toxicity. Comprehensive research over the past 20 years has indicated that resveratrol has beneficial effects against a wide range of diseases, such as cardiovascular, neurological, metabolic and liver autoimmune diseases. Various lines of evidence indicate that resveratrol possesses antioxidant, anti-inflammatory, antibacterial and antisenescence activities. It is a key factor in multiple intracellular signaling pathways and targets various signaling molecules involved in tumor progression. It also inhibits signaling molecules involved in cancer invasion, such
A

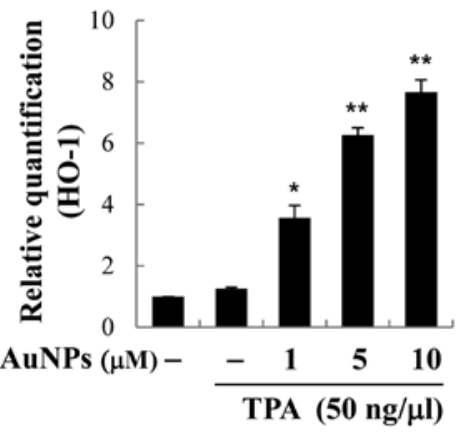

B

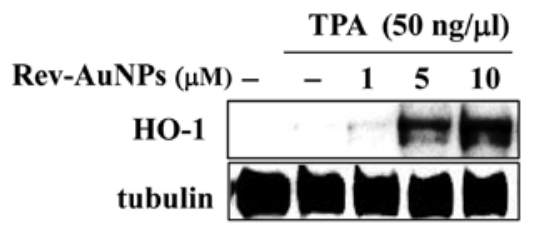

C

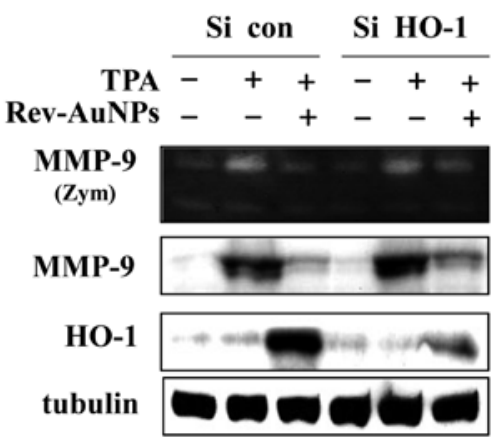

Figure 6. HO-1 siRNA diminishes the anti-invasion effect of gold-conjugated resveratrol nanoparticles (Rev-AuNPs) in TPA-stimulated breast cancer cells. (A) MCF-7 cells were cultured with increasing concentrations of Rev-AuNPs for 4 h. HO-1 mRNA expression was determined by real-time PCR. Each bar represents the mean \pm SE of 3 independent experiments in each group; ${ }^{*} \mathrm{P}<0.05$ and ${ }^{* *} \mathrm{P}<0.01$ vs. control-treated group. (B) Cells were incubated for $12 \mathrm{~h}$ with the indicated concentration of Rev-AuNPs. Total cellular extracts were prepared, and western blotting was performed. (C) Cells were transfected with control siRNA and $\mathrm{HO}-1$ siRNA using X-tremeGENE according to the manufacturer's instructions. The cells were treated with RevAuNPs $(10 \mu \mathrm{M})$ for $1 \mathrm{~h}$ and stimulated with TPA for $24 \mathrm{~h}$, and the activity of MMP-9 was examined by gelatin zymography.

as MMP-9, COX-2, NF- $\mathrm{kB}$ and AP-1 (9,10,22). Importantly, the capacity of resveratrol to suppress tumor proliferation and metastasis in a number of cancer cell lines has provoked increased attention for its use as an anticancer agent. For this reason, resveratrol has received a great deal of attention and the use of preparations containing resveratrol is increasing. Alternative pharmacological techniques are necessary to maximize the efficiency of resveratrol by improving its targeting and bioavailability $(22,23)$.

Recently, considerable research has focused on the use of nanoscale materials as new effective anticancer agents in humans. Specifically, AuNPs have been developed as an important tool for medical applications. The biological synthesis of AuNPs is eco-friendly and an ideal method to develop environmentally sustainable nanoparticles. The bioconjugation of AuNPs is widely used to develop biocompatible AuNPs for medical applications $(16,20,24)$. In the present study, resveratrol was used as a reductant for the green synthesis of Rev-AuNPs and its invasive efficacy on MCF-7 


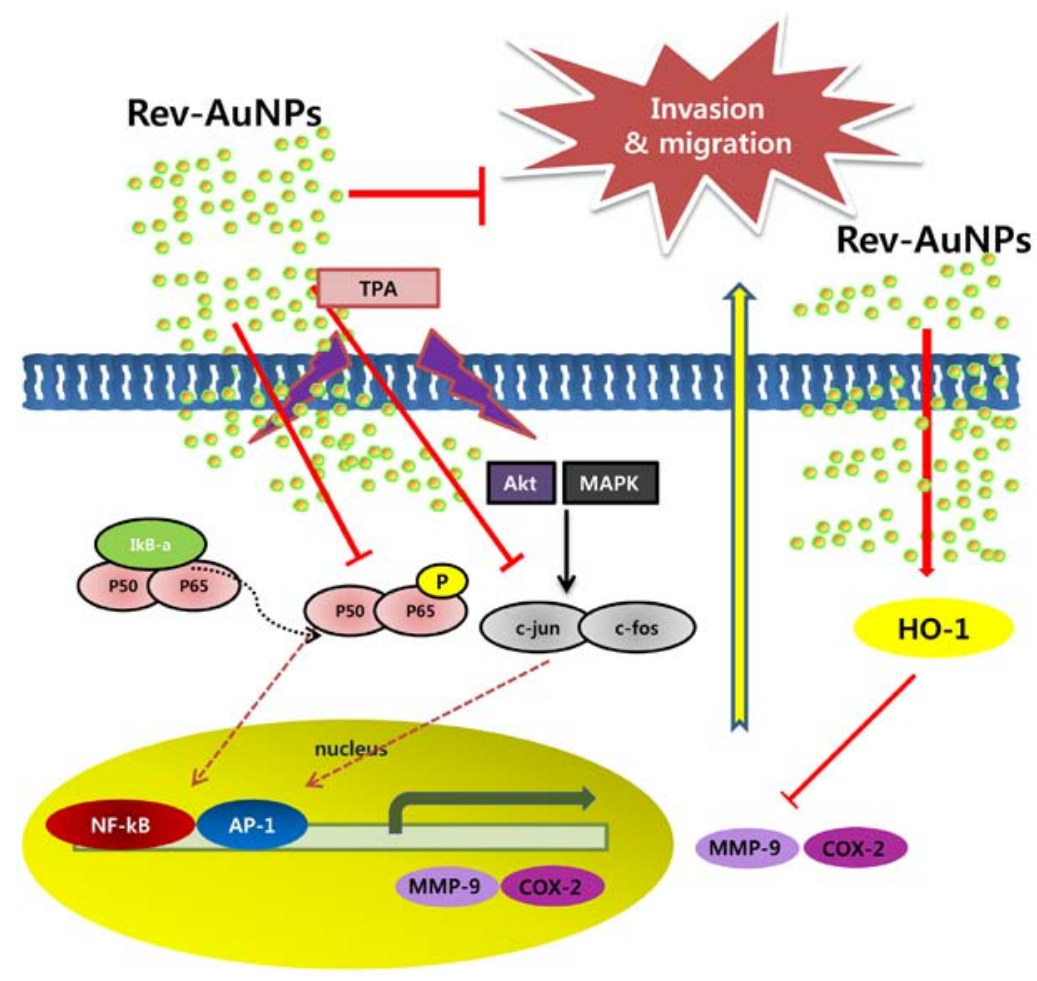

Figure 7. Mechanism involved in the anti-invasive activity of the gold-conjugated resveratrol nanoparticles (Rev-AuNPs). Rev-AuNPs exhibited anti-invasive activity by inhibiting the activation of NF- $\mathrm{KB}$ and AP-1, by inhibiting the phosphorylation of Akt and ERK, and by inducing HO-1.

breast cancer cells was examined. Spectroscopic, light scattering, and microscopic techniques were used to characterize the Rev-AuNPs, including a UV-visible spectrum analysis, DLS and TEM. Using DLS, we observed that the particle size and surface charge (zeta potential) of Rev-AuNPs were $30.75 \pm 3.41 \mathrm{~nm}$ and $-32.8 \pm 2.47 \mathrm{mV}$. Using TEM, we determined the particle size distribution as well as the morphology of the Rev-AuNPs; their size was $22.28 \pm 2.98 \mathrm{~nm}$.

The invasion and metastasis of breast cancer cells are essential biological characteristics of tumor progression and most cancer deaths are due to metastasis development. Thus, the regulation or even regression of the process of metastasis would be a significant advance in the treatment of breast cancer. TPA as a protein kinase activator is frequently used as a biomedical research tool in models of carcinogenesis (2). In the present study, we systematically investigated the effect of Rev-AuNPs on TPA-stimulated breast cancer cells by applying several capable experimental methods. First, we discovered that TPA induced migration and invasion activity in the breast cancer cells, and in the presence of Rev-AuNPs, the TPA-induced migration and invasion activity decreased. The extracellular matrix regulates multiple cellular functions necessary for tumor invasion and extracellular matrix components (i.e., MMPs) are key mediators of this progression. Activation of MMP-9 is correlated with increased cell invasion, angiogenesis, as well as aggressive breast cancer. A specific inhibitor of MMP-9 is TIMP-1 and MMP-2 is regulated by TIMP-2; accordingly, the elucidation of TIMP-1 and TIMP-2 regulation will provide insight into the process of metastasis $(2,3)$. We analyzed the inhibitory effect of Rev-AuNPs on MMP-9 activation in breast cancer cells. The Rev-AuNPs inhibited MMP-9 not by inhibiting MMP-9 enzymatic activity, but by the suppression of MMP-9 mRNA and protein expression, whereas the activation of MMP-2, TIMP-1 and TIMP-2 was not affected. Furthermore, we found that Rev-AuNPs inhibited TPA-induced expression of COX-2 at the mRNA and protein levels in the MCF-7 breast cancer cells.

To further investigate the anti-invasive mechanism of Rev-AuNPs, we examined the effects of Rev-AuNPs on NF- $\kappa$ B and AP-1 signaling. The NF- $\mathrm{B}$ and AP-1 signaling pathways are essential for cell migration, survival and proliferation and are associated with tumor progression (6). Indeed, we found that Rev-AuNPs significantly inhibited the activation of NF-kB and AP-1 in TPA-stimulated breast cancer cells. PI3K/Akt and MAPKs are closely connected with tumor progression (4). Several key molecules involved in invasion and metastasis, such as MMPs and COX-2, are also regulated by PI3K/Akt and MAPK signaling. We were further interested in the inhibitory mechanism of Rev-AuNPs on PI3K/Akt and MAPK, which regulate a range of biological processes implicated in tumor progression. PI3K/Akt and ERK play roles in the regulation of various cancers. We compared the anti-invasive effect between resveratrol and Rev-AuNPs in MCF-7 breast cancer cells. Notably, Rev-AuNPs exhibited a better anti-invasive activity than resveratrol in most parts, and did not exhibit cytotoxicity. The inhibitory effect of Rev-AuNPs on MMP-9, COX-2, $\mathrm{NF}-\kappa \mathrm{B}, \mathrm{AP}-1, \mathrm{PI} 3 \mathrm{~K} / \mathrm{Ak}$ and ERK activation was stronger than that of resveratrol for the same concentrations.

Several beneficial effects of HO-1, including antioxidant, anti-inflammation and antisenescence activity, have been described. Overexpression of HO-1 may decrease tumor progression via decreasing the range of intracellular oxidants. However, conflicting reports have also indicated that the induction of HO-1 is associated with tumor progression and is 
also correlated with anticancer drug resistance. Nevertheless, many anticancer drug candidates have been reported to prevent tumor progression via the induction of HO-1 $(21,25,26)$. We investigated whether Rev-AuNPs inhibit TPA-induced MMP-9 activation in MCF-7 breast cancer cells via HO-1 expression. Rev-AuNP-mediated inhibition of MMP-9 activity was also suppressed by the knockdown of endogenous HO-1 in the MCF-7 breast cancer cells.

In conclusion, resveratrol was used as a reductant for the green synthesis of Rev-AuNPs. The synthesized Rev-AuNPs were completely characterized with respect to particle size, shape, and surface charge by spectroscopic, light scattering and microscopic techniques. We found that Rev-AuNPs effectively inhibited breast cancer cell progression. This inhibition involved many molecules, including MMP-9, COX-2, NF- $\mathrm{BB}, \mathrm{AP}-1, \mathrm{PI} 3 \mathrm{~K} / \mathrm{Akt}$ and ERK (Fig. 7). Although further investigation is needed to clarify the precise mechanisms by which Rev-AuNPs inhibit breast cancer progression and to identify the Rev-AuNPs responsible for the observed effects, Rev-AuNPs may be considered a potential therapeutic agent for the treatment of breast cancer. As well, our results that focus on nanoscale materials as new effective anticancer agents and clinical utilization are demonstrated, concluding with considerations to develop AuNPs for clinical applications.

\section{Acknowledgements}

The present study was supported by the Basic Science Research Program through the National Research Foundation of Korea (NRF) funded by the Ministry of Education, Science and Technology (2015R1D1A1A01059450). The present study was financially supported by the Ministry of Trade, Industry and Energy (MOTIE) and Korea Institute for Advancement of Technology (KIAT) through the Promoting Regional Major Industry (R0004422).

\section{References}

1. Gnerlich JL, Deshpande AD, Jeffe DB, Seelam S, Kimbuende E and Margenthaler JA: Poorer survival outcomes for male breast cancer compared with female breast cancer may be attributable to in-stage migration. Ann Surg Oncol 18: 1837-1844, 2011

2. Bertolini F: Adipose tissue and breast cancer progression: A link between metabolism and cancer. Breast 22 (Suppl 2): S48-S49, 2013.

3. Song J, Su H, Zhou YY and Guo LL: Prognostic value of matrix metalloproteinase 9 expression in breast cancer patients: A metaanalysis. Asian Pac J Cancer Prev 14: 1615-1621, 2013.

4. Yang S and Han H: Effect of cycloxygenase-2 silencing on the malignant biological behavior of MCF-7 breast cancer cells Oncol Lett 8: 1628-1634, 2014.

5. Jin ML, Park SY, Kim YH, Park G and Lee SJ: Halofuginone induces the apoptosis of breast cancer cells and inhibits migration via downregulation of matrix metalloproteinase-9. Int J Oncol 44: 309-318, 2014.

6. Liao YF, Rao YK and Tzeng YM: Aqueous extract of Anisomeles indica and its purified compound exerts anti-metastatic activity through inhibition of NF- $\kappa \mathrm{B} / \mathrm{AP}-1$-dependent MMP-9 activation in human breast cancer MCF-7 cells. Food Chem Toxicol 50: 2930-2936, 2012.

7. Kim YS, Sull JW and Sung HJ: Suppressing effect of resveratrol on the migration and invasion of human metastatic lung and cervical cancer cells. Mol Biol Rep 39: 8709-8716, 2012.

8. Chakraborty A, Gupta N, Ghosh K and Roy P: In vitro evaluation of the cytotoxic, anti-proliferative and anti-oxidant properties of pterostilbene isolated from Pterocarpus marsupium. Toxicol In Vitro 24: 1215-1228, 2010.
9. Harikumar KB, Kunnumakkara AB, Sethi G, Diagaradjane P, Anand P, Pandey MK, Gelovani J, Krishnan S, Guha S and Aggarwal BB: Resveratrol, a multitargeted agent, can enhance antitumor activity of gemcitabine in vitro and in orthotopic mouse model of human pancreatic cancer. Int J Cancer 127: 257-268, 2010

10. Amri A, Chaumeil JC, Sfar S and Charrueau C: Administration of resveratrol: What formulation solutions to bioavailability limitations? J Control Release 158: 182-193, 2012.

11. Pangeni R, Sahni JK, Ali J, Sharma S and Baboota S: Resveratrol: Review on therapeutic potential and recent advances in drug delivery. Expert Opin Drug Deliv 11: 1285-1298, 2014.

12. Nicol JR, Dixon D and Coulter JA: Gold nanoparticle surface functionalization: A necessary requirement in the development of novel nanotherapeutics. Nanomedicine 10: 1315-1326, 2015.

13. Park $\mathrm{H}$, Tsutsumi $\mathrm{H}$ and Mihara $\mathrm{H}$ : Cell penetration and cell-selective drug delivery using $\alpha$-helix peptides conjugated with gold nanoparticles. Biomaterials 34: 4872-4879, 2013.

14. Karuppaiya P, Satheeshkumar E, Chao WT, Kao LY, Chen EC and Tsay HS: Anti-metastatic activity of biologically synthesized gold nanoparticles on human fibrosarcoma cell line HT-1080. Colloids Surf B Biointerfaces 110: 163-170, 2013.

15. Suganya KS, Govindaraju K, Kumar VG, Dhas TS, Karthick V, Singaravelu G and Elanchezhiyan M: Blue green alga mediated synthesis of gold nanoparticles and its antibacterial efficacy against Gram positive organisms. Mater Sci Eng C 47: 351-356, 2015.

16. Sanna V, Pala N, Dessì G, Manconi P, Mariani A, Dedola S, Rassu M, Crosio C, Iaccarino C and Sechi M: Single-step green synthesis and characterization of gold-conjugated polyphenol nanoparticles with antioxidant and biological activities. Int J Nanomedicine 9: 4935-4951, 2014.

17. Kondath S, Srinivas Raghavan B, Anantanarayanan R and Rajaram R: Synthesis and characterisation of morin reduced gold nanoparticles and its cytotoxicity in MCF-7 cells. Chem Biol Interact 224C: 78-88, 2014.

18. Barathmanikanth S, Kalishwaralal K, Sriram M, Pandian SR, Youn HS, Eom S and Gurunathan S: Anti-oxidant effect of gold nanoparticles restrains hyperglycemic conditions in diabetic mice. J Nanobiotechnology 8: 16, 2010.

19. Khan HA, Abdelhalim MA, Alhomida AS and Al Ayed MS: Transient increase in IL-1 $\beta$, IL- 6 and TNF- $\alpha$ gene expression in rat liver exposed to gold nanoparticles. Genet Mol Res 12: 5851-5857, 2013.

20. Kumar CG, Poornachandra Y and Mamidyala SK: Green synthesis of bacterial gold nanoparticles conjugated to resveratrol as delivery vehicles. Colloids Surf B Biointerfaces 123: 311-317, 2014.

21. Chao CY, Lii CK, Hsu YT, Lu CY, Liu KL, Li CC and Chen HW: Induction of heme oxygenase-1 and inhibition of TPA-induced matrix metalloproteinase-9 expression by andrographolide in MCF-7 human breast cancer cells. Carcinogenesis 34: 1843-1851, 2013.

22. Lee CW, Yen FL, Huang HW, Wu TH, Ko HH, Tzeng WS and Lin CC: Resveratrol nanoparticle system improves dissolution properties and enhances the hepatoprotective effect of resveratrol through antioxidant and anti-inflammatory pathways. J Agric Food Chem 60: 4662-4671, 2012.

23. Li J, Wang X, Wang C, Chen B, Dai Y, Zhang R, Song M, Lv G and Fu D: The enhancement effect of gold nanoparticles in drug delivery and as biomarkers of drug-resistant cancer cells. ChemMedChem 2: 374-378, 2007.

24. Correard F, Maximova K, Estève MA, Villard C, Roy M, Al-Kattan A, Sentis M, Gingras M, Kabashin AV and Braguer D: Gold nanoparticles prepared by laser ablation in aqueous biocompatible solutions: Assessment of safety and biological identity for nanomedicine applications. Int J Nanomedicine 9: 5415-5430, 2014.

25. Lee WY, Chen YC, Shih CM, Lin CM, Cheng CH, Chen KC and Lin CW: The induction of heme oxygenase-1 suppresses heat shock protein 90 and the proliferation of human breast cancer cells through its byproduct carbon monoxide. Toxicol Appl Pharmacol 274: 55-62, 2014.

26. Lin CW, Shen SC, Hou WC, Yang LY and Chen YC: Heme oxygenase-1 inhibits breast cancer invasion via suppressing the expression of matrix metalloproteinase-9. Mol Cancer Ther 7: 1195-1206, 2008. 